\title{
Comments on Shuzhen Li et al. "Systematic review of patellar resurfacing in total knee arthroplasty"
}

\author{
Gerold Labek
}

Received: 16 March 2011 /Accepted: 23 April 2011 /Published online: 20 May 2011

(C) Springer-Verlag 2011

\section{Dear Editor,}

The article by Shuzhen et al. is an excellent review on a controversial issue following active guidelines for assessment of scientific papers [1]. However, some limitations from a methodological point of view should be taken into account.

Prospective, randomised, trials are considered to be the gold standard in study design. The main goal of these studies is to eliminate or control confounders by the patient and the surgeon by blinding the patient and the person assessing the endpoint concerning the treatment arm in the study. This works quite well in pharmaceutical studies, when white pills in white packages are administered.

This concept has some limitations in surgical procedures, particularly when it comes to implantation of a medical device and the endpoint is revision surgery. To fulfill the basic criteria for randomised controlled trials (RCTs), neither the patient, nor the surgeon deciding on a revision surgery, must have access to any information as to whether the patient has received a patellar button or not. This includes access to patient files and X-rays. I wonder if any ethics review board would agree on a concept in which additional surgery must be performed without proper planning and assessment for the indication? I am not aware of any explanation of how to overcome that situation in any of the publications included in that review.

This might be an explanation for the inconsistent findings concerning revision rate and clinical outcome.

G. Labek ( $($ )

Department of Orthopaedic Surgery,

Innsbruck Medical University,

Anichstrasse 35,

6020 Innsbruck, Austria

e-mail: gerold.labek@efort.org
Large cohort studies based on arthroplasty registers (including all patients in a country excluding all clinicalstudy-related confounders) show no statistically significant difference between patients who received a patella resurfacing and those who did not [2]. Taking data from the RCTs analysed serious and transferable to the population, one should expect almost double the revision rate after total knee arthroplasty (TKA) if no patella resurfacing was done. For such a huge effect, surgeons in general do not require studies to make that observation. Why is there no consensus in patient treatment worldwide? Why is there no supporting data in other studies on TKA not focused on patella resurfacing? Why do register data and other large observational studies not confirm the finding in RCTs?

I think we should be aware that the endpoint "revision rate" is highly dependent on subjective decisions, and the total number of revision cases is quite low in all RCTs published. Revision rate is a major indicator for outcome but should be in line with clinical scores to support objective decision-making processes in the cohorts analysed [2]. I also think we should be aware that confounders appearing in conformity in the majority of individual studies can bias the conclusion, even if the review methodology is properly conducted.

\section{Best Regards}

Gerold Labek

\section{References}

1. Shuzhen L, Yueping C, Wei S, Jinmin Z, Shinqing H, Xiangping L (2011) Systematic review of patellar resurfacing in total knee arthroplasty. Int Orthop 35:305-316

2. Labek G, Liebensteiner M, Williams A, Thaler M, Stöckl B (2010) Impact of patellar resurfacing on the revision rate in total knee arthroplasty. Eur Orthop Traumatol 1:95-101 